\title{
Surfaces with Constant Chebyshev Angle II
}

\author{
Carlos M. C. RIVEROS and Armando M. V. CORRO
}

Universidade de Brasília and Universidade Federal de Goiâs

(Communicated by M. Guest)

\begin{abstract}
In this paper we classify a class of surfaces with negative Gaussian curvature parametrized by a generalized Chebyshev net with constant Chebyshev angle in the Euclidean 3-space. As an application we obtain for each constant Chebyshev angle a seven-parameter family of complete surfaces.
\end{abstract}

\section{Introduction}

Bianchi in [1]-[2] studies a class of surfaces with negative Gaussian curvature obtained by generalizing Bäcklund transformation for surfaces with constant negative Gaussian curvature. Fujioka in [6] introduces the notion of generalized Chebyshev nets which is a natural generalization of Chebyshev nets for surfaces with constant negative Gaussian curvature and shows that a Bianchi surface with constant Chebyshev angle parametrized by a generalized Chebyshev net is a piece of a right helicoid; in this case the Chebyshev angle is $\pi / 2$.

Riveros and Corro in [7] obtained a characterization for a class of surfaces with a generalized Chebyshev net and constant Chebyshev angle different from $\pi / 2$. The characterization is obtained by showing that the coefficients of the first and second fundamental form of these surfaces depend on a meromorphic function which satisfies a certain differential equation. The characterization is based on the results obtained in [3], [4] and [5].

In this work, we classify a class of surfaces with negative Gaussian curvature parametrized by a generalized Chebyshev net with constant Chebyshev angle. We also study the completeness of such surfaces.

\section{Prelimiminaries}

In the following we consider only surfaces with negative Gaussian curvature in the Euclidean 3-space $\mathbf{R}^{3}$. Since such a surface has two directions, called the asymptotic directions,

Received May 7, 2012; revised August 16, 2012 Mathematics Subject Classification: 30D30, 53A10, 35C99

Key words and phrases: Liouville equation, Chebyshev net, asymptotic lines

The first author was partially supported by PRONEX-FAPDF and the second author was partially supported by CNPq, CAPES and PROCAD. 
in which the normal curvature vanishes, we can parametrize the surface locally by asymptotic line coordinates $(x, y)$ :

$$
\chi: \Omega \subset \mathbf{R}^{2} \rightarrow \mathbf{R}^{3}
$$

If the Gaussian curvature is $-\frac{1}{\rho^{2}}$ for a positive function $\rho$ on $\Omega$ then the fundamental forms become as follows:

$$
I=A^{2} d x^{2}+2 A B \cos \varphi d x d y+B^{2} d y^{2}, \quad I I=\frac{2 A B \sin \varphi}{\rho} d x d y,
$$

where $A=\left|\chi_{x}\right|, \quad B=\left|\chi_{y}\right|$ and $\varphi$ is the angle between the asymptotic lines, called the Chebyshev angle. Changing the coordinates if necessary, we may assume that $0<\varphi<\pi$.

DEFINITION 1. A parametrization of a surface is called a generalized Chebyshev net if $A=B$.

REMARK 1. In this paper the inner product is defined by $\langle\rangle:, \mathbf{C} \times \mathbf{C} \rightarrow \mathbf{R}$. In the computation we use the following properties: If $f, g: \mathbf{C} \rightarrow \mathbf{C}$ are holomorphic functions of $z=x+i y \in \mathbf{C}$ then

$$
\langle f, g\rangle_{x}=\left\langle f^{\prime}, g\right\rangle+\left\langle f, g^{\prime}\right\rangle, \quad\langle f, g\rangle_{y}=\left\langle i f^{\prime}, g\right\rangle+\left\langle f, i g^{\prime}\right\rangle .
$$

The following result, obtained in [7], characterizes the surfaces in $\mathbf{R}^{3}$ with a generalized Chebyshev net, negative Gaussian curvature and constant Chebyshev angle $\varphi \neq \pi / 2$.

THEOREM 1. Let $M \subset \mathbf{R}^{3}$ be a connected orientable Riemann surface and $\varphi$ a constant different from $\pi / 2$. There exists an immersion $X: M \rightarrow \mathbf{R}^{3}$ with a generalized Chebyshev net, negative Gaussian curvature $K=-\frac{1}{e^{2 u}}$ and Chebyshev angle $\varphi$ if and only if there exists a global meromorphic function $h: M \rightarrow \mathbf{C}$ such that $h^{\prime}(z) \neq 0$ at all regular points and it admits only simple poles, satisfying the following

$$
\begin{aligned}
& 2 e^{c}(1+\varepsilon \cos \varphi)\left\langle h,(1+\varepsilon i) h^{\prime}\right\rangle\left\langle h^{\prime}, h^{\prime}\right\rangle \\
& \quad-\left[1+e^{c}(1+\varepsilon \cos \varphi)|h|^{2}\right]\left\langle h^{\prime},(1+\varepsilon i) h^{\prime \prime}\right\rangle=0 .
\end{aligned}
$$

Moreover, locally the fundamental forms of $X$ are given by

$$
\begin{aligned}
I & =e^{(1-\varepsilon \cos \varphi) u+c}\left[d x^{2}+2 \cos \varphi d x d y+d y^{2}\right], \\
I I & =2 e^{-\varepsilon \cos \varphi u+c} \sin \varphi d x d y,
\end{aligned}
$$

where

$$
u(x, y)=\log \left(\frac{1+e^{c}(1+\varepsilon \cos \varphi)|h(z)|^{2}}{2\left|h^{\prime}(z)\right|}\right)^{\frac{2}{1+\varepsilon \cos \varphi}}
$$

$c \in \mathbf{R}, z=x+i y \in \mathbf{C}, \varepsilon= \pm 1$. 
LEMMA 1. If $f, g, h: \mathbf{C} \rightarrow \mathbf{C}$ are holomorphic functions of $z=x+i y \in \mathbf{C}$ such that

$$
\langle 1, f\rangle+|h|^{2}\langle 1, g\rangle=0
$$

Then

$$
f=-\bar{z}_{1} h+i c_{2}, \quad g=i c_{1}+\frac{z_{1}}{h},
$$

where $c_{i}$ are real constants and $z_{1} \in \mathbf{C}$.

ProOF. The equation (5) can be written as

$$
P=\langle 1, f\rangle+\langle h, h g\rangle=0 .
$$

From this equation, it follows that $P_{x x}+P_{y y}=0$, i.e.

$$
\left\langle h^{\prime},(h g)^{\prime}\right\rangle=0,
$$

therefore $(h g)^{\prime}=i c_{1} h^{\prime}, c_{1} \in \mathbf{R}$ and by integration we get

$$
h g=i c_{1} h+z_{1}, \quad z_{1} \in \mathbf{C} .
$$

Substituting (8) into (7), we obtain

$$
\langle 1, f\rangle+\left\langle h, z_{1}\right\rangle=0 .
$$

Differentiating with respect to $x$ and $y$ respectively, we get

$$
\left\langle 1, f^{\prime}\right\rangle+\left\langle h^{\prime}, z_{1}\right\rangle=0, \quad\left\langle 1, i f^{\prime}\right\rangle+\left\langle i h^{\prime}, z_{1}\right\rangle=0 .
$$

From these equations we have

$$
\overline{f^{\prime}}+z_{1} \overline{h^{\prime}}=0
$$

and consequently

$$
\frac{f^{\prime}}{h^{\prime}}=-\overline{z_{1}}
$$

Integrating, we get

$$
f=-\bar{z}_{1} h+z_{2}, \quad z_{2} \in \mathbf{C},
$$

substituting this expression into (9), we obtain $\left\langle 1, z_{2}\right\rangle=0$, it follows from this that $z_{2}=$ $i c_{2}, c_{2} \in \mathbf{R}$, consequently we obtain the expression of $f$ given in (6). The expression of $g$ is obtained from equation (8).

\section{Main Results}

The following Theorem classifies a class of surfaces in $\mathbf{R}^{3}$ with a generalized Chebyshev net, negative Gaussian curvature and constant Chebyshev angle $\varphi \neq \pi / 2$. 
THEOREM 2. Let $M \subset \mathbf{R}^{3}$ be a connected orientable Riemann surface and $\varphi$ a constant different from $\pi / 2 . X: M \rightarrow \mathbf{R}^{3}$ is an immersion with a generalized Chebyshev net, negative Gaussian curvature $K=-\frac{1}{e^{2 u}}$ and Chebyshev angle $\varphi$ if and only if the global meromorphic function $h: M \rightarrow \mathbf{C}$ of the Theorem 1 is given by:

1)

$$
h(z)=\frac{i}{\bar{z}_{1}}\left[S \operatorname{coth}\left(\frac{\varepsilon+i}{4} S z-z_{0}\right)+c_{2}\right], \quad \text { in this case } M=\mathbf{C}-\left\{\alpha_{k}\right\}_{k \in \mathbf{Z}},
$$

$\alpha_{k}=\frac{2(\varepsilon-i)}{S} \operatorname{Re}\left(z_{0}\right)+\frac{2(1+\varepsilon i)}{S}\left[\operatorname{Im}\left(z_{0}\right)+k \pi\right], \quad S=\sqrt{c_{2}^{2}+\frac{\left|z_{1}\right|^{2}}{e^{c}(1+\varepsilon \cos \varphi)}}, \quad c, c_{2} \in \mathbf{R}$, $z_{0}, z_{1} \in \mathbf{C}, \quad \varepsilon= \pm 1$

2)

$$
h(z)=e^{(\varepsilon+i) \frac{c_{2}}{2} z+z_{2}}, \quad \text { in this case } M=\mathbf{C}, \quad c_{2} \neq 0, \quad z_{2} \in \mathbf{C}, \quad \varepsilon= \pm 1 .
$$

PROOF. From Theorem 1, the proof of this Theorem reduces to determining all of the solutions of the differential equation (1).

The equation (1) can be written as

$$
\left\langle 1, \frac{b h^{\prime \prime}}{h^{\prime}}\right\rangle+|h|^{2}\left\langle 1, \frac{a b h^{\prime \prime}}{h^{\prime}}-\frac{2 a b h^{\prime}}{h}\right\rangle=0,
$$

where $a=e^{c}(1+\varepsilon \cos \varphi), \quad b=1+\varepsilon i, \varepsilon= \pm 1$.

From Lemma 1, we obtain

$$
\begin{gathered}
\frac{b h^{\prime \prime}}{h^{\prime}}=-\bar{z}_{1} h+i c_{2}, \\
\frac{a b h^{\prime \prime}}{h^{\prime}}-2 a b \frac{h^{\prime}}{h}=i c_{1}+\frac{z_{1}}{h} .
\end{gathered}
$$

Substituting (13) into (14) we get

$$
a\left(-\bar{z}_{1} h+i c_{2}\right)-2 a b \frac{h^{\prime}}{h}=i c_{1}+\frac{z_{1}}{h}
$$

and consequently

$$
b h^{\prime}=-\frac{\bar{z}_{1}}{2} h^{2}+\frac{i}{2}\left(c_{2}-\frac{c_{1}}{a}\right) h-\frac{z_{1}}{2 a} .
$$

Differentiating (15) and dividing by $h^{\prime}$, we have that

$$
\frac{b h^{\prime \prime}}{h^{\prime}}=-\bar{z}_{1} h+\frac{i}{2}\left(c_{2}-\frac{c_{1}}{a}\right) \text {. }
$$


From (13) and (16), it follows that $c_{1}=-a c_{2}$, substituting this expression into (15), we get

$$
h^{\prime}=-\frac{\bar{z}_{1}}{2 b} h^{2}+\frac{i c_{2}}{b} h-\frac{z_{1}}{2 a b} .
$$

Now we will determine the solutions of the equation (17).

Supposing that $z_{1} \neq 0, c_{2} \in \mathbf{R}$ and substituting the expressions $w_{1}=-\frac{\bar{z}_{1}}{2 b}, w_{2}=$ $\frac{i c_{2}}{b}, w_{3}=-\frac{z_{1}}{2 a b}$ into (17), we obtain

$$
h^{\prime}=w_{1}\left[\left(h+\frac{w_{2}}{2 w_{1}}\right)^{2}-\frac{w_{2}^{2}-4 w_{1} w_{3}}{4 w_{1}^{2}}\right] .
$$

On the other hand

$$
\frac{w_{2}^{2}-4 w_{1} w_{3}}{4 w_{1}^{2}}=-\frac{1}{\bar{z}_{1}^{2}}\left(c_{2}^{2}+\frac{\left|z_{1}\right|^{2}}{a}\right)
$$

Putting $S^{2}=c_{2}^{2}+\frac{\left|z_{1}\right|^{2}}{a}$, from (18) and (19), we get

$$
w_{1}=\frac{h^{\prime}}{\left[\left(h+\frac{w_{2}}{2 w_{1}}\right)^{2}+\frac{1}{\bar{z}_{1}^{2}} S^{2}\right]} \text {. }
$$

We can show that

$$
\frac{h^{\prime}}{\left[\left(h+\frac{w_{2}}{2 w_{1}}\right)^{2}+\frac{1}{\bar{z}_{1}^{2}} S^{2}\right]}=\frac{h^{\prime}}{\frac{i}{\bar{z}_{1}} S}\left[\frac{\frac{1}{2}}{h+\frac{w_{2}}{2 w_{1}}-\frac{i}{\bar{z}_{1}} S}-\frac{\frac{1}{2}}{h+\frac{w_{2}}{2 w_{1}}+\frac{i}{\bar{z}_{1}} S}\right] .
$$

Using (21) in (20), we get

$$
\frac{i}{\bar{z}_{1}} S w_{1} d z=\frac{\frac{1}{2} d h}{h+\frac{w_{2}}{2 w_{1}}-\frac{i}{\bar{z}_{1}} S}-\frac{\frac{1}{2} d h}{h+\frac{w_{2}}{2 w_{1}}+\frac{i}{\bar{z}_{1}} S} .
$$

Integrating

$$
\frac{i}{\bar{z}_{1}} S w_{1} z+z_{0}=\frac{1}{2} \log \left(h+\frac{w_{2}}{2 w_{1}}-\frac{i}{\bar{z}_{1}} S\right)-\frac{1}{2} \log \left(h+\frac{w_{2}}{2 w_{1}}+\frac{i}{\bar{z}_{1}} S\right) .
$$

Hence,

$$
e^{\frac{2 i}{z_{1}} S w_{1} z+2 z_{0}}=\frac{h+\frac{w_{2}}{2 w_{1}}-\frac{i}{\bar{z}_{1}} S}{h+\frac{w_{2}}{2 w_{1}}+\frac{i}{\bar{z}_{1}} S}
$$


Solving for $h$, we get

$$
h(z)=\frac{i}{\bar{z}_{1}} S\left(\frac{1+e^{\frac{2 i}{z_{1}} S w_{1} z+2 z_{0}}}{1-e^{\frac{2 i}{z_{1}} S w_{1} z+2 z_{0}}}\right)-\frac{w_{2}}{2 w_{1}} .
$$

Substituting the expressions $w_{1}=-\frac{\bar{z}_{1}}{2 b}, \quad w_{2}=\frac{i c_{2}}{b}, b=1+\varepsilon i$ into (23), we have that the solutions of equation (17) are given by

$$
h(z)=\frac{i}{\bar{z}_{1}}\left[S\left(\frac{1+e^{-\frac{\varepsilon+i}{2} S z+2 z_{0}}}{1-e^{-\frac{\varepsilon+i}{2} S z+2 z_{0}}}\right)+c_{2}\right],
$$

which is equivalent to equation (10). We observe that $\sinh \left(\frac{\varepsilon+i}{4} S z-z_{0}\right)=0$ if and only if $z=\alpha_{k}$.

For the case $z_{1}=0, c_{2} \neq 0$, the equation (17) reduces to $h^{\prime}=\frac{i c_{2}}{b} h$.

Integrating, we obtain

$$
\log h=\frac{i c_{2}}{b} z+z_{2}, \quad z_{2} \in \mathbf{C}
$$

Hence, $h(z)=e^{(\varepsilon+i) \frac{c_{2}}{2} z+z_{2}}$, is a solution to the equation (17) given in (11). Which concludes the proof of Theorem 2 .

COROLLARY 1. There exists a seven-parameter family of surfaces with a generalized Chebyshev net and constant Chebyshev angle $\varphi \neq \pi / 2$ whose first and second fundamental forms are given by

$$
\begin{aligned}
I= & e^{c}\left(\frac { \sqrt { 2 } | \operatorname { s i n h } ( \frac { \varepsilon + i } { 4 } S z - z _ { 0 } ) | ^ { 2 } } { | \overline { z } _ { 1 } | S ^ { 2 } } \left\{\left|\bar{z}_{1}\right|^{2}+e^{c}(1+\varepsilon \cos \varphi)\right.\right. \\
& \left.\left.\times\left|S \operatorname{coth}\left(\frac{\varepsilon+i}{4} S z-z_{0}\right)+c_{2}\right|^{2}\right\}\right)^{\frac{2(1-\varepsilon \cos \varphi)}{1+\varepsilon \cos \varphi}}\left[d x^{2}+2 \cos \varphi d x d y+d y^{2}\right] \\
I I= & 2 e^{c}\left(\frac { \sqrt { 2 } | \operatorname { s i n h } ( \frac { \varepsilon + i } { 4 } S z - z _ { 0 } ) | ^ { 2 } } { | \overline { z } _ { 1 } | S ^ { 2 } } \left\{\left|\bar{z}_{1}\right|^{2}+e^{c}(1+\varepsilon \cos \varphi)\right.\right. \\
& \left.\left.\times\left|S \operatorname{coth}\left(\frac{\varepsilon+i}{4} S z-z_{0}\right)+c_{2}\right|^{2}\right\}\right)^{\frac{-2 \varepsilon \cos \varphi}{1+\varepsilon \cos \varphi}} \sin \varphi d x d y .
\end{aligned}
$$

Moreover, if $c_{2}=0$ and $c \geq-\log (1+\varepsilon \cos \varphi)$ then the surfaces defined by (24) and (25) are complete.

PROOF. From Theorem 2, it follows that for the meromorphic function given by (10) there exists a seven-parameter family $X: \mathbf{C}-\left\{z_{k}\right\}_{k \in \mathbf{Z}} \rightarrow \mathbf{R}^{3}$ of surfaces with a generalized 
Chebyshev net and constant Chebyshev angle. Moreover, we have that

$$
h^{\prime}(z)=\frac{(1-\varepsilon i) S^{2}}{4 \bar{z}_{1} \sinh ^{2}\left(\frac{\varepsilon+i}{4} S z-z_{0}\right)} .
$$

Substituting (10) and (26) into (4), we get

$$
\begin{aligned}
u(x, y)= & \log \left(\frac { \sqrt { 2 } | \operatorname { s i n h } ( \frac { \varepsilon + i } { 4 } S z - z _ { 0 } ) | ^ { 2 } } { | \overline { z } _ { 1 } | S ^ { 2 } } \left\{\left|\bar{z}_{1}\right|^{2}+e^{c}(1+\varepsilon \cos \varphi)\right.\right. \\
& \left.\left.\times\left|S \operatorname{coth}\left(\frac{\varepsilon+i}{4} S z-z_{0}\right)+c_{2}\right|\right\}\right)^{\frac{2}{1+\varepsilon \cos \varphi}} .
\end{aligned}
$$

Substituting (27) in (2) and (3), we obtain the equations (24) and (25). On the other hand, if $c_{2}=0$ and $c \geq-\log (1+\varepsilon \cos \varphi)$, we can show that

$$
\left(\frac{\sqrt{2}|\sinh R|^{2}}{\left|\bar{z}_{1}\right| S^{2}}\left\{\left|\bar{z}_{1}\right|^{2}+e^{c}(1+\varepsilon \cos \varphi)|S \operatorname{coth} R|^{2}\right\}\right)^{\frac{2(1-\varepsilon \cos \varphi)}{1+\varepsilon \cos \varphi}} \geq\left(\frac{\sqrt{2}}{\left|z_{1}\right|}\right)^{\frac{2(1-\varepsilon \cos \varphi)}{1+\varepsilon \cos \varphi}}
$$

where, $R=\frac{\varepsilon+i}{4} S z-z_{0}$. In fact, from the condition $c \geq-\log (1+\varepsilon \cos \varphi)$, we have

$$
\begin{gathered}
\frac{\sqrt{2}|\sinh R|^{2}}{\left|\bar{z}_{1}\right| S^{2}}\left\{\left|\bar{z}_{1}\right|^{2}+e^{c}(1+\varepsilon \cos \varphi)|S \operatorname{coth} R|^{2}\right\} \geq \frac{\sqrt{2}|\sinh R|^{2}}{\left|\bar{z}_{1}\right| S^{2}}\left\{\left|\bar{z}_{1}\right|^{2}+S^{2}|\operatorname{coth} R|^{2}\right\} \\
=\frac{\sqrt{2}}{\left|\bar{z}_{1}\right| S^{2}}\left(\left|\bar{z}_{1}\right|^{2}|\sinh R|^{2}+\frac{\left|z_{1}\right|^{2}}{e^{c}(1+\varepsilon \cos \varphi)}|\cosh R|^{2}\right) \\
=\frac{\sqrt{2}\left|\bar{z}_{1}\right|}{S^{2} e^{c}(1+\varepsilon \cos \varphi)}\left(e^{c}(1+\varepsilon \cos \varphi)|\sinh R|^{2}+|\cosh R|^{2}\right) \geq \frac{\sqrt{2}}{\left|z_{1}\right|}
\end{gathered}
$$

Therefore, the equation (28), is an consequence of this inequality. From (28), it follows that

$$
d s^{2} \geq C d \bar{s}^{2}
$$

where,

$$
\begin{aligned}
& \begin{aligned}
d s^{2}= & e^{c}\left(\frac { \sqrt { 2 } | \operatorname { s i n h } ( \frac { \varepsilon + i } { 4 } S z - z _ { 0 } ) | ^ { 2 } } { | \overline { z } _ { 1 } | S ^ { 2 } } \left\{\left|\bar{z}_{1}\right|^{2}+e^{c}(1+\varepsilon \cos \varphi)\right.\right. \\
& \left.\left.\times\left|S \operatorname{coth}\left(\frac{\varepsilon+i}{4} S z-z_{0}\right)\right|^{2}\right\}\right)^{\frac{2(1-\varepsilon \cos \varphi)}{1+\varepsilon \cos \varphi}}\left[d x^{2}+2 \cos \varphi d x d y+d y^{2}\right] \\
d \bar{s}^{2}= & d x^{2}+2 \cos \varphi d x d y+d y^{2}
\end{aligned} \\
& \text { and } C=e^{c}\left(\frac{\sqrt{2}}{\left|z_{1}\right|}\right)^{\frac{2(1-\varepsilon \cos \varphi)}{1+\varepsilon \cos \varphi}} .
\end{aligned}
$$


Since the metric $C d \bar{s}^{2}$ is complete, it follows that the metric $d s^{2}$ also is complete. On the other hand, when $z \rightarrow \alpha_{k}$, we have that $R \rightarrow k \pi i$ and the metric also satisfies the equation (29) and therefore is complete. This concludes the proof of Corollary 1.

REMARK 2. Considering the solution $h(z)=e^{(\varepsilon+i) \frac{c_{2}}{2} z+z_{2}}$, given in Theorem 2, we obtain the family of the surfaces obtained in Corollary 2 in [7].

\section{References}

[ 1 ] L. Bianchi, Sopra alcume nuove classi di superficie e di sistemi tripli ortogonali, Ann. Mat. (2) 18 (1890), 301-358.

[2] L. BIANCHI, Lezioni di geometria differenziale, Pisa (1909).

[ 3 ] F. BRito, J. HouniE and M. L. Leite, Liouville's formula in arbitrary planar domains, Nonlinear Analysis (60) (2005), 1287-1302.

[4] F. BRito and M. L. Leite, Uniqueness and globality of the Liouville formula for entire solutions of $\frac{\partial^{2} \log \lambda}{\partial z \partial \bar{z}}+\frac{\lambda}{2}=0$, Arch. Math. 80 (2003), 501-506.

[ 5 ] F. BRito, M. L. Leite and V. Neto, Liouville's formula under the viewpoint of minimal surfaces, Commun. Pure Appl. Anal. 3(1) (2004), 41-51.

[ 6 ] A. FujiokA, Bianchi surfaces with constant Chebyshev angle, Tokyo J. Math. 27, No. 1 (2004), 149-153.

[ 7 ] C. M. C. Riveros and A. M. V. Corro, Surfaces with constant Chebyshev angle, Tokyo J. Math. 35, No. 2 (2012), 359-366.

Present Addresses:

Carlos M. C. Riveros

DEPARTAMENTO DE MATEMÁtica,

UNIVERSIDADE DE BRASÍLIA,

BRASÍLIA-DF, 70910-900 BRAZIL.

e-mail: carlos@mat.unb.br

ARMAndo M. V. CORRO

INSTITUTO DE MATEMÁTICA E EstatísTICA,

UNIVERSIDAdE FEDERAL DE GoIÂs,

GOIANIA-GO, 74001-970 BRAZIL.

e-mail: corro@mat.ufg.br 